\title{
MERIDIAN OBSERVATIONS AT SANTIAGO, CHILE
}

\author{
C. Anguita, G. Carrasco, P. Loyola, V. N. ŠıšKina and M.S. Zverev
}

Observations of the Southern Reference Star (SRS) and Bright Star (BS) programmes were started in January 1963 with the Repsold meridian circle of the Santiago Observatory. Up to July 1, 1967, a total of 86725 star observations arranged in 1520 series had been observed.

In order to investigate the instrumental system, series of fundamental stars between $+41^{\circ}$ and $-69^{\circ} \mathrm{SP}$ were included in the observing programme. A total of 214 such series were observed, 123 being with 'clamp East' and 91 with 'clamp West'. The number of individual star observations in these 214 series was 7344 . These observations were reduced using Bessel's formula

$$
\alpha=T+(u+m)+n \tan \delta+c^{\prime} \sec \delta,
$$

where $u$ denotes the clock correction and $c^{\prime}$ includes the collimation error, diurnal aberration and the contact width. The value of $n$ which best fits the observations between declinations $+30^{\circ}$ and $-30^{\circ}$ was calculated and denoted by $n_{e}$. For stars outside this zone, $n$ was calculated from the expression

$$
n=\left\{(\alpha-T)-(\overline{\alpha-T})_{e}\right\} /\left(\tan \delta-\overline{\tan \delta_{e}}\right)
$$

and $\Delta n=\left(n-n_{e}\right)$ formed.

The differences $\Delta n$ were grouped by zones of declination $5^{\circ}$ wide and the mean value $\overline{\Delta n}$ calculated for each zone. The dependence of $\overline{\Delta n}$ on declination was determined graphically. In order to reduce the scatter, the sliding means between two neighbouring groups were plotted. The results for 'clamp East' and 'clamp West' are shown separately in Figure 1, the first curve depending on 2068 values and the second on 1762 .

In order to investigate the dependence of $\overline{\Delta n}$ on right ascension, the observations were divided into four zones, each 6 hours wide and centered at $0,6,12$ and 18 hours. The dependence of $\overline{\Delta n}$ on declination is shown, separately for each of these four right ascension zones, in Table 1. The systematic nature of the values given in this table suggest the need of corrections to the FK4 of the type $\Delta \alpha_{\delta}$ and $\Delta \alpha_{\alpha}$. The values of $\frac{1}{2}\left(\Delta n+\Delta n_{s p}\right)$ and $\frac{1}{2}\left(\Delta n-\Delta n_{s p}\right)$ are given in Table 2 . The former may be used to estimate the $\Delta \alpha_{\alpha}$ errors in FK4 and the latter errors of the type $\Delta \alpha_{\delta}$ (Zverev, 1965).

The fact that the implied systematic errors are practically the same for both positions of the instrument makes it appear unlikely that we are dealing with an instrumental

Perek (ed.), Highlights of Astronomy, 292-296. (C) I.A.U. 
MERIDIAN OBSERVATIONS AT SANTIAGO, CHILE

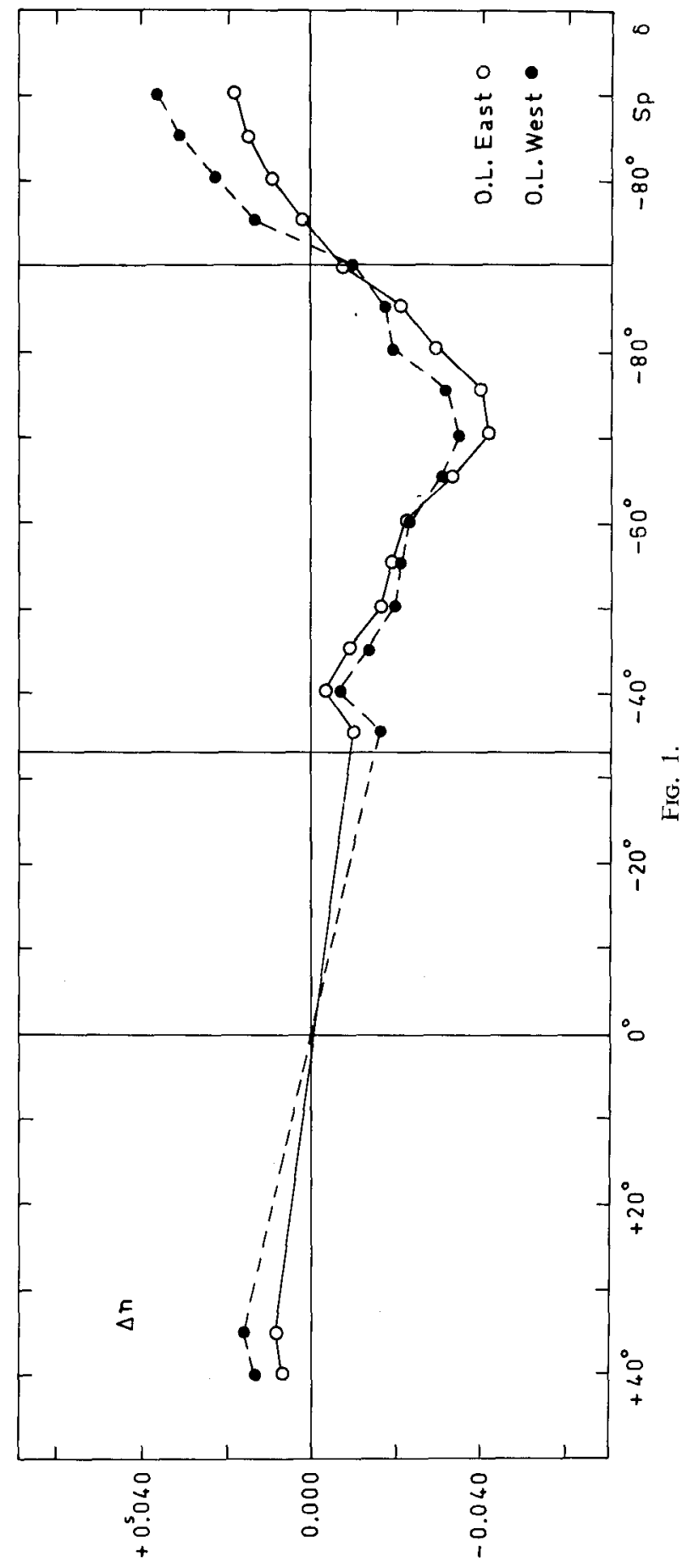


Table 1

$$
\Delta n_{(\mathrm{E}+\mathrm{W})}(0.001)
$$

$\begin{array}{lc}\delta \backslash \alpha & 0^{\mathrm{h}} \\ +40^{\circ} & +20.8 \\ +35^{\circ} & +27.0 \\ & \\ -35^{\circ} & -18.1 \\ -40^{\circ} & -02 \cdot 8 \\ -45^{\circ} & -13 \cdot 2 \\ -50^{\circ} & -21.9 \\ -55^{\circ} & -20.3 \\ -60^{\circ} & -32.0 \\ -65^{\circ} & -37.5 \\ -70^{\circ} & -41 \cdot 2 \\ -75^{\circ} & -49.6 \\ -80^{\circ} & -39.5 \\ -85^{\circ} & -20.8 \\ -90^{\circ} & -05.6 \\ -85^{\circ} \mathrm{sp} & +03.4 \\ -80^{\circ} \mathrm{sp} & +06.8 \\ -75^{\circ} \mathrm{sp} & +22.6 \\ -70^{\circ} \mathrm{sp} & +31.0\end{array}$

Numbers of Series

Numbers of $\Delta n$ $\alpha_{M}$

$\begin{aligned} & 6^{\mathrm{h}} \\ & \\ &+ 04 \cdot 4 \\ &+ 06 \cdot 1 \\ & \\ &-12 \cdot 7 \\ &-08 \cdot 7 \\ &-15 \cdot 2 \\ &-16 \cdot 7 \\ &-22 \cdot 0 \\ &-22 \cdot 6 \\ &-29 \cdot 8 \\ &-36 \cdot 7 \\ &-37 \cdot 7 \\ &-24 \cdot 0 \\ &-18.9 \\ &-04 \cdot 9 \\ &+13 \cdot 6 \\ &+18 \cdot 4 \\ &+21 \cdot 7 \\ &+27 \cdot 0\end{aligned}$

75

1413

06 ' 4

$$
\begin{aligned}
& 1^{\mathrm{h}} \\
& +07.7 \\
& +13 \cdot 1 \\
& -14.4 \\
& -03 \cdot 4 \\
& -08 \cdot 5 \\
& -23 \cdot 2 \\
& -21 \cdot 0 \\
& -18.6 \\
& -30 \cdot 0 \\
& -38.4 \\
& -32 \cdot 1 \\
& -19.0 \\
& -20.5 \\
& -10.8 \\
& +04.8 \\
& +17.1 \\
& +23 \cdot 2 \\
& +25.7
\end{aligned}
$$

65

1272

$11^{\text {. }} 4$ $18^{h}$

$+06.3$

$+08.7$

$-07.9$

$-04.0$

$-04.5$

$-09.2$

$-19.5$

$-33.2$

$-41.1$

$-42 \cdot 8$

$-29.5$

$-20.6$

$-23.3$

$-09.6$

$-01.8$

$+08.9$

$+22 \cdot 3$

$+43.8$

27

546

17 h 7

\section{Table 2}

$$
\begin{array}{lllll}
\frac{1}{2}\left(\Delta n+\Delta n_{s p}\right) & 0.001 \\
\overline{\mathrm{E}+\mathrm{W}} & 00 \mathrm{~h} 1 & 06 ! 4 & 11^{\mathrm{h}} 4 & 17 ! 7
\end{array}
$$

$\begin{array}{cccccccc}\delta & \text { E } & \text { W } & \overline{\mathrm{E}+\mathrm{W}} & 00^{\mathrm{h}} .1 & 06 \text { h. } & 11^{\mathrm{h}} 4 & 17^{\mathrm{h}} .7 \\ 90^{\circ} & -07.4 & -08.6 & -08.0 & -05.6 & -04.9 & -10.8 & -09.6 \\ 85^{\circ} & -09.8 & -02.7 & -06.6 & -08.7 & -02.6 & -07.8 & -12.6 \\ 80^{\circ} & -10.0 & +01.6 & -04.8 & -16.4 & -02.8 & -01.0 & -05.8 \\ 75^{\circ} & -13.0 & -00.2 & -07.0 & -13.5 & -08.0 & -04.4 & -03.6 \\ 70^{\circ} & -12.0 & +00.1 & -06.2 & -05.1 & -04.8 & -06.4 & +00.5\end{array}$

Mean $\quad-10.4 \quad-02.0 \quad-06.5 \quad-09.9 \quad-04.6 \quad-06.1 \quad-06.2$

$$
\frac{1}{2}\left(\Delta n-\Delta n_{s p}\right) 0^{s} .001
$$

$\begin{array}{cccccccccc}\delta & \mathrm{E} & \mathrm{W} & \overline{\mathrm{E}+\mathrm{W}} & 00 \mathrm{~h} .1 & 06.4 & 11.4 & 17 \mathrm{~h} .7 & \text { Mean } & \Delta \alpha_{\delta} \cos \delta \\ 90^{\circ} & 0 & 0 & 0 & 0 & 0 & 0 & 0 & 0 & 0 \\ 85^{\circ} & -11.5 & -15.4 & -13.3 & -12.1 & -16.2 & -12.6 & -10.8 & -12.9 & -12.9 \\ 80^{\circ} & -18.8 & -21.2 & -19.2 & -23.2 & -21.2 & -18.0 & -14.8 & -19.3 & -19.0 \\ 75^{\circ} & -27.6 & -31.2 & -29.2 & -36.1 & -29.7 & -27.6 & -25.9 & -29.8 & -28.8 \\ 70^{\circ} & -29.5 & -35.4 & -32.4 & -36.1 & -31.8 & -32.0 & -43.3 & -35.8 & -33.6\end{array}$




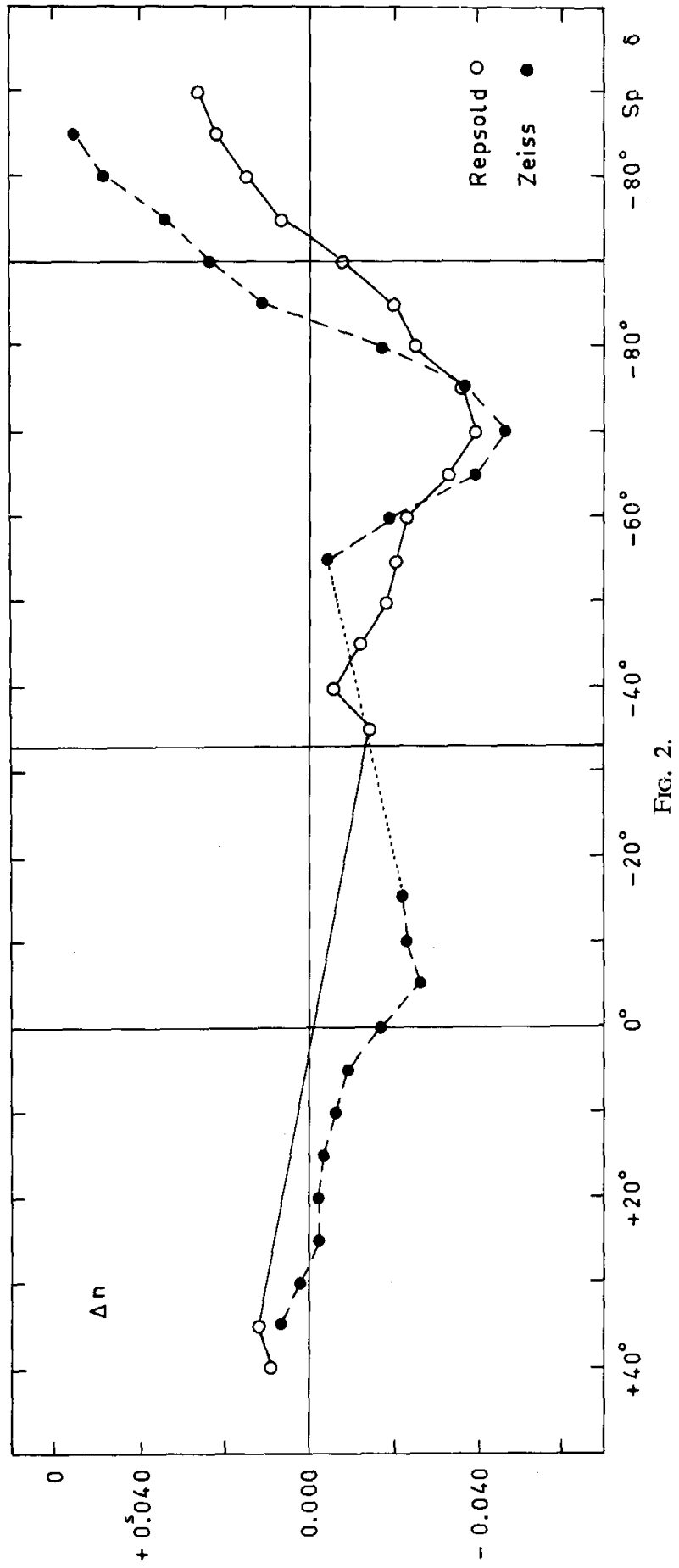


peculiarity. This can be further checked, however, by 41 series of observations made between April 1963 and September 1964 with a Zeiss broken transit.

The azimuth of this instrument was determined from star $i$ by the expression

$$
a_{i}=\left\{(\overline{(\alpha-T})_{\bar{z}}-(\alpha-T)_{i}\right\} /\left(\bar{A}_{\bar{z}}-A_{i}\right)
$$

where $\overline{(\alpha-T)}_{\bar{z}}$ is the mean difference between the right ascension and transit time for stars near the zenith, the time being already corrected for contact width, level and diurnal aberration. $A_{\bar{z}}$ and $A_{i}$ denote the azimuth coefficients in Mayer's formula. A total of 1372 azimuth determinations were obtained for this instrument. The mean azimuth $a_{N}$ found from stars near declination $+30^{\circ}$ was used as a reference azimuth and individual differences $\Delta a$ were calculated from

$$
\Delta a_{i}=a_{N}-a_{i}
$$

As in the case of the meridian circle observations, mean values of $\Delta a$ were formed for zones of declination $5^{\circ}$ in width. These mean values were transformed into $\Delta n$ using the relation

$$
\Delta n=b \sin \phi-\Delta a \cos \phi,
$$

where $b$ is the level error and $\phi$ the latitude. But as the level error has already been applied, the relation reduces to

$$
\Delta n=-0.835 \Delta a
$$

The resulting values of $\Delta n$ are plotted in Figure 2 , in which the $\frac{1}{2(E+\mathrm{W})}$ values of $\Delta n$ obtained from the Repsold meridian circle are also plotted. There is very good agreement between the values of $\Delta n$ obtained with the two instruments which seems to indicate that there is a real systematic error of the type $\Delta \alpha_{\delta}$ in FK4.

\section{Reference}

Zverev, M.S. (1965) Astr. Zu., 42, No. 4. 